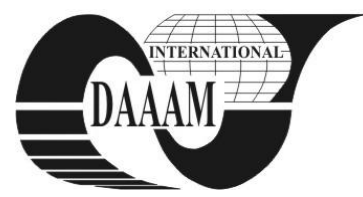

\title{
DEVICE FOR MEASURING SPECTRAL - SPATIAL DISTRIBUTION OF LIGHT DISPERSED BY SURFACES
}

\section{LETUNOV, A[lexander] A[lexeevich]; BARLADIAN, B[oris]; GALAKTIONOV, V[ladimir] A[lexandrovich]; ERSHOV, S[ergey] V[alentinovich]; VOLOBOY, A[lexey] \& ZUEVA, E[lena]}

\begin{abstract}
Device for measuring spectral - spatial distribution of light dispersed by surfaces is presented. These data are necessary for calculations and computer simulation of reflectors, liquid crystal displays, clothes, paints, and other objects and materials with non trivial light-scattering characteristics. The modern systems of computer graphics also need such data for accurate simulation of light propagation. The direct measurements are the more reliable methods to obtain these data, but this task is not easy.

Key words: Computer graphics, physically realistic image, $B R D F$, light scattering, non-uniform grid, high-lighted zone
\end{abstract}

\section{INTRODUCTION}

The general way to describe light scattering behavior of a surface is Bidirectional Reflection/ Transmittance Distribution Function BRDF/BTDF. Defined in every point of the surface, this function shows what portion of the light energy received from a direction $\mathrm{K}_{\mathrm{i}}$, will be reflected/ transmitted by the surface in another direction $\mathrm{K}_{\mathrm{s}}$. For isotropic surface, it is a function of four variables - three spatial (one angle is necessary to define an incident beam and two angles to define a direction of view), and one spectral. It is usually presented in table form, with uniform or non-uniform grid of input data. BRDF/BTDF measurement is a rather sophisticated task. The main difficulties are a great volume of data and great difference in values of the function and its gradient in the different areas.

In the paper we describe the concept of BRDF/BTDF measurements, developed by authors, the device (laboratory installation) which realizes this concept, and some results of its exploitation. Near the normal direction of input beam, the angle distribution is transformed into the space one and is registered as an image. For the other directions, the fiber optics is used. For both cases, a CCD-camera is used as a registering device.

This paper is the improved version of the works (Letunov, 1999; Voloboy, 2006). In the given version the accuracy of measurements is higher due to the better image detector - a monochrome digital video camera with 16-bit encoder.

\section{CONSTRUCTION OF THE DEVICE}

The device should minimize the measurement time and produce the reliable results, sufficient for practical aims. Usually reflection/ transmittance function has a high gradient for the viewing angles near the direction of specular reflection or direct transmittance. The method of measurements and the construction of the device must provide both the high angle resolution in these areas, and the inclusion of the total space angle of scattering. There are some works, where digital cameras are used for imaging all direction of diffusion simultaneously. In the paper (Ward, 1992) the space is everted with the aids of reflecting or diffusing semi-sphere. Camera is placed near the sample and a wide-angle "fisheye" objective is used. However, this decision leads to a high contribution of parasitic diffusion from the inner elements of semi-sphere into the registered signal. It decreases the reliability of the results.

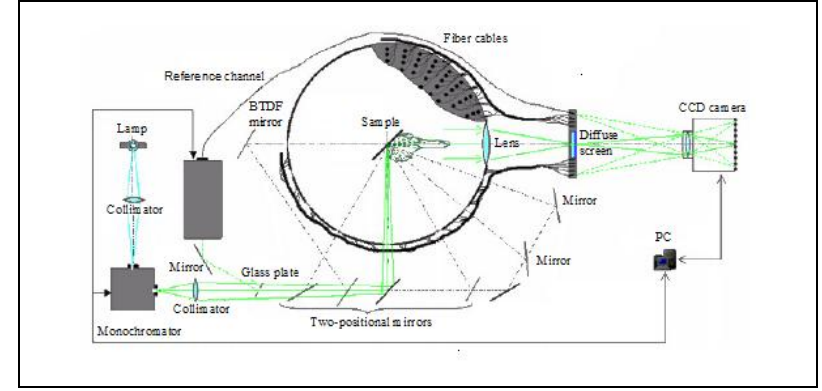

Fig. 1. The general scheme of the device

To vary spatial arguments (two spatial angles) we can use either a motion of some parts of construction, or the numerous fixed canals of light propagation. In our case, the incident angle is varied via the motion of the sample, and the total area of reflected light directions is covered by a number of fibers fixed in the space by a special way. The light dispersed by the sample surface from the area of maximal resolution is gathered via lens, and from the other directions - via fibers. Camera is placed separately from the sample and "looks" at the screen, in the center of which the light passed through the lens is concentrated on the mate plate, and the output ends of fibers are disposed around it. The light, dispersing by the sample, is measured for numerous ( 400) directions simultaneously.

As a support for fibers, a semi-sphere with internal radius $200 \mathrm{~mm}$ is used. It has several holes for incident light and 430 holes for fibers (only 150 of them are actually used now). The inputs ends of fibers are directed to the center of the semisphere, where the sample is placed.

The sample is lighted by almost parallel beam in the narrow spectral interval. A xenon arc lamp is used as a light source. The central fragment of the arc is projected via a lens with double magnification onto the input of monochromator, controlled by computer. The monochromator scans the range from 390 to 730 $\mathrm{nm}$, subdividing it into 35 spectral fragments with the $10 \mathrm{~nm}$ width. The range and the width may be chosen by different way, depending on the goal of the measurements and the available information about filters transmittance. It is possible to increase /decrease the range and to make it non uniform. In our case the violet end was restricted by the decreasing of camera resolution with wavelength decreasing, and the red end by available passport data. The whole visible area of the specter was covered.

As an output collimator, we used a lens with focal length $\mathrm{F}=150 \mathrm{Mm}$, focal plane near to the plane of the sample and zooming $\sim 10$. This lens transfers the image of the output of the monochromator. Some part of a light flow is directed into a reference channel, with the aid of a glass parallel-sided plate. The plate is posed under the angle $\sim 6^{\circ}$ to the beam and derives about $8 \%$ of a light flow. Then a computer controlled wheel with a number of certificated neutral filters is placed for the dynamical range increasing. The light passed throughout the plate is reflected by the system of mirrors and reaches the sample via one of input holes at the equator of semi-sphere.

The incident beam lies in horizontal plane. The sample is in vertical plane, the center of lighted area of the sample coincides 
with the center of semi-sphere. The sample has two angle degrees of freedom, about vertical and horizontal (in the sample plane) axis. For an angle of incidence determination, the rotation about vertical axis is sufficient; the second degree of freedom is used for adjustment. For anisotropic samples the additional facility is used, which allows to rotate the sample about its normal raising from the center $\mathrm{O}$.

For reflection measurements, to provide a needed incident angle, a beam may pass through one of five holes in the equator of the sphere. For transmittance measurements, the beam comes to sphere from the back side, where there is no wall. The system of mirrors provides this possibility.

The direction of distributed light $\mathrm{K}_{\mathrm{s}}$ is determined by two angles $\theta$ и $\phi$. The angle $\theta$, is formed by $K_{\mathrm{s}}$ and the axe $Z$ (the direction of specular reflection or refraction). The angle $\phi \square$ is the angle between the incident plane and the plane $\left(\mathrm{K}_{\mathrm{s}}, \mathrm{Z}\right)$, fig.2. The angle $\sigma$ between incident beam and the normal to the surface gives the third coordinate. This coordinate system is used in computer graphics and luminance simulation (Ignatenko and all, Barladian and all). It makes it convenient to use the measured functions as input data for such systems.

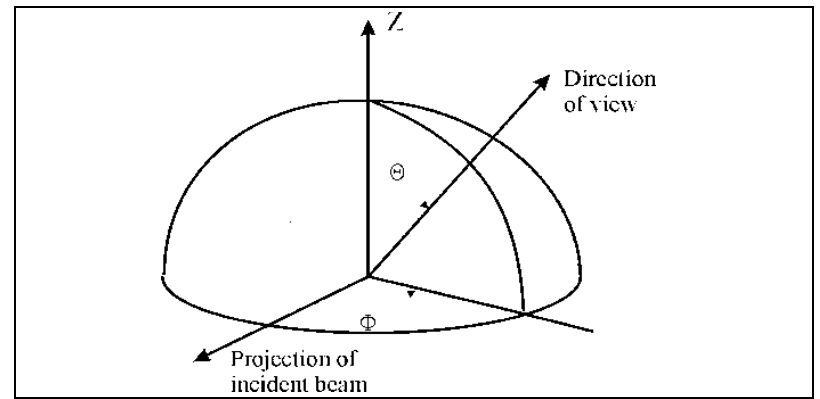

Fig.2. The angles defining a view direction

To realize this system the axe $\mathrm{Z}$ should be directed to the camera permanently. We use the stable camera. The required disposition of axes is obtained due to synchronous change the direction of incident beam and the sample rotation.

The optic fibers are disposed on the sphere so that the output angles $\theta, \phi$ form the needed non-uniform grid. The lens with diameter $80 \mathrm{~mm}$ and focal length $150 \mathrm{Mm}$ gathers the light, dispersed by the sample within $10^{\circ}$ from the direction of specular reflection, and focuses it on a think round plate made from mate glass, placed in the center of rectangular screen. The objective of camera is located in the place, where the image of sample surface could focus if there would no mate plate. It is a zone of maximal luminance.

The mate plate is disposed in focal plane of the lens. For this reason the angular distribution of the light, reflected from the sample surface, is transformed into the spatial one. The values of $\theta$ and $\phi$ are transformed into the corresponding values of linear coordinates on the screen. It means that the light, reflected at any point of the sample surface in the direction defined by the angles $\theta, \phi$ and gathered by the lens, will come to the same point on the plate. It allows improving the angle resolution near the direction of specular reflection.

At the same screen the output ends of fibers are placed, including reference channel fiber. The side of the screen turned to the camera is a fragment of the sphere with the center in the camera objective. The camera produces the image of the screen with the mate plate and the ends of fibers. The semi-transparent mate screen allows, at first, to make the portions of light coming from the different fragments of the surface viewed under the same angle proportional to its brightness. Secondly, it decreases the contrast of the brightness between lens and fiber's parts of the image.

For amplitude calibration of the device, the diffusing certificated sample is necessary. We have the standard for normal incident direction only. The calibration process includes the total measurements cycle for all wavelengths for the standard, placed normally to incident beam. This data are stored and then used for all working measurements.

\section{PROCESS OF MEASUREMENTS. RESULTS}

In the measurement cycle, the arguments of BRDF/BTDF functions are scanned in the following order: angles of viewing, the wavelengths, incident angles. For the given wavelength and the established incident light angle, the device does register simultaneously all BRDF/BTDF allowed by its construction. The time of measurements practically does not depend on the number of fibers used in the experiment. For the fixed direction of incident beam, the scanning of spectral component is done automatically, with the aid of monochromator, controlled by computer. To change the angle of incident beam, the manual operations are necessary. For light transmittance measurements, it is the sample turning. For light reflection measurements, additionally it is necessary to change the direction of incident beam synchronously with the sample turning.

The device was successively used for practical tasks and demonstrated the following characteristics. The dynamic range of brightness coefficient (BRDF/BTDF): $0.05-2500$. Reproducibility of results:1-3\%. Accuracy: 4-8\%. Angle resolution: $0.5^{\circ}-1.5^{\circ}$ at the area $<10^{\circ}$ from specular reflection direction, $1.5^{\circ}-8^{\circ}$ for the other directions.

\section{CONCLUSIONS.}

Due to original author's concept, the efficient device for BDF measurements was created. This concept includes two ideas. For high-lighted zone the image is formed by lens, so that the angle distribution is transformed into the space one. For ordinary scattered light we use fiber optics.

The need of BDF measurements is proved by numerous demands during all the period of probe exploitation of the device. Encouraged by this fact, we plan some modifications. As it was mentioned, the measurement cycle is not fully automated, the incident direction is restricted by semi-sphere, certificated standard exists only for normal directions. All these restrictions should be overcome in new version of the device.

\section{ACKNOWLEDGEMENTS}

This work was supported by the Russian Leading Scientific Schools Foundation, project NSh- 8129.2010.9, RFBR, grants 09-01-00299, 10-01-00302 and 11-01-00870, and by INTEGRA Inc.

\section{REFERENCES}

Letunov A.A, Barladian B.H., Zueva E.Yu., Veshnevetc V., Soldatov S. CCD-based device for BRDF measurements in computer graphics. The 9th International Conference on Computer Graphics \& Vision. Russia, Moscow, 1999, pp.129-135

Ward G.J., Measuring and Modeling Anisotropic Reflection. Computer Graphics, Volume 26, Number 2, July 1992

Ignatenko A., Barladian B., Dmitriev K., Ershov S., Galaktionov V., Valiev I., Voloboy A., A Real-Time 3D Rendering System with BRDF Materials and Natural Lighting. The 14-th International Conference on Computer Graphics and Vision GraphiCon-2004, Moscow, September 6-11, 2004. Conference Proceedings, pp. 159-162

Barladian B.H., Voloboy A.G., Vyukova N.I., Galactionov V.A, Deriabin N.B. The lighting simulation and photorealistic image synthesis using Internet technologies. "Programming", № 5, 2005

Voloboy A.G., Galactionov V.A., Ershov S.V., Letunov A.A., Potemin I.S. The device-software complex for lightscattering behavior of surface measurements. Information technologies and computational systems, 2006. № 4. 